\title{
GO-modified membranes for vanadium redox flow battery
}

\author{
Saidatul Sophia ${ }^{1,2}$, Ebrahim Abouzari Lotf ${ }^{2,3,}$, , Arshad Ahmad ${ }^{2,3}$, Pooria Moozarm Nia ${ }^{2}$, \\ and Roshafima Rasit Ali ${ }^{1,2}$ \\ ${ }^{1}$ Malaysia-Japan International Institute of Technology, Universiti Teknologi Malaysia, Jalan \\ Sultan Yahya Petra, 54100 Kuala Lumpur, Malaysia . \\ ${ }^{2}$ Center of Hydrogen Energy, Institute of Future Energy, Universiti Teknologi Malaysia, 81310 \\ Johor Bahru, Johor, Malaysia . \\ ${ }^{3}$ Department of Chemical Engineering, School of Chemical and Energy Engineering, Faculty of \\ Engineering, Universiti Teknologi Malaysia, 81310 Johor Bahru, Johor, Malaysia.
}

\begin{abstract}
Graphene oxide (GO) has attracted tremendous attention in membrane-based separation field as it can filter ions and molecules. Recently, GO-based materials have emerged as excellent modifiers for vanadium redox flow battery (VRFB) application. Its high mechanical and chemical stability, nearly frictionless surface, high flexibility, and low cost make GO-based materials as proper materials for the membranes in VRFB. In VRFB, a membrane acts as the key component to determine the performance. Therefore, employing low vanadium ion permeability with excellent stability membrane in vanadium electrolytes is important to ensure high battery performance. Herein, recent progress of GO-modified membranes for VRFB is briefly reviewed. This review begins with current membranes used for VRFB, followed by the challenges faced by the membranes. In addition, the transport mechanism of vanadium ion and the stability properties of GO-modified membranes are also discussed to enlighten the role of GO in the modified membranes.
\end{abstract}

Keywords: Graphene oxide, membrane, ion selectivity, vanadium redox flow battery

\section{Introduction}

In recent years, there has been a growing interest in the development of electrochemical energy storage (EES) devices that convert chemical reaction to electrical energy, and store and release the energy when demanded $[1,2]$. Among all EES devices, vanadium redox flow battery (VRFB) has gained attraction as a promising candidate in tackling the issues related to energy storage, especially for large-scale applications. VRFB has

\footnotetext{
*Corresponding author: ebrahim@utm.com
} 
attracted great concern among users due to its capability to minimise severe crosscontamination between different elements. Unlike other types of EES devices, VRFB uses all vanadium states $\left(\mathrm{V}^{2+}, \mathrm{V}^{3+}, \mathrm{V}^{4+}\right.$, and $\left.\mathrm{V}^{5+}\right)$ in both positive and negative electrolytes. Additionally, due to the presence of the same elements in both electrolytes, VRFB also offers other interesting advantages such as long life cycle, deep discharge capacity, and low environmental impact [3-6].

In VRFB, ion exchange membrane (IEM) plays a significant role that regulates the efficiency of battery. The main role of IEM in VRFB is to prevent diffusion of active elements in the positive and negative electrolytes and simultaneously maintain the neutrality of electrolytes by transporting protons and sulphates [7]. Low vanadium permeability, high proton conductivity, outstanding chemical and mechanical stability, as well as low cost are key characteristics for a VRFB membrane [8,9].

Vanadium permeability is one of the fundamental measurements that needs to be considered when selecting membranes for VRFBs and predicting the efficiency of battery. Vanadium permeability determines the Coulombic efficiency (CE) and capacity loss of battery over several charge-discharge cycles that are often caused by the unequal permeability rates of vanadium ions across the membranes. Knowledge of vanadium ion transfer across the membrane can be useful in order to optimise VRFB operating conditions, as well as to reduce vanadium permeability $[8,9]$.

Regarding membrane stability, highly acidic electrolytes in VRFB should be considered. Although it has been reported that perfluorinated sulfonic acid (PFSA) membranes (such as Nafion) can withstand harsh electrolyte conditions, the high cost of membrane is the main challenge for material developers. According to the literature by Minke and Turek [10], the cost of the membranes for VRFB contributes up to $50 \%$ of the whole cost system. Hence, various efforts have been done towards finding alternative materials for more economical membranes with better efficiency.

Recently, graphene oxide (GO)-based membranes have gained attraction as alternative materials for VRFB application. GO-based membrane is not something new in the separation field since it has been widely employed in various applications such as vapour transport [11], selective gas transport [12], hydrogen separation [13], water transport [14], and proton exchange [15]. Although GO-based materials have been employed in various fields, its application as a membrane material is still new [16-19]. Due to its excellent mechanical stability, good resistance to strong acidic and alkaline solvents, flexibility, and low-cost, GO is a carbon-based material that exhibits remarkable potential in phase separation [20-22]. Hence, the current paper reviews recently published works on employing GO-based membranes in VRFB application and its potential improvement. Figure 1 shows the number of articles published regarding GO-modified membrane for VRFB applications. 


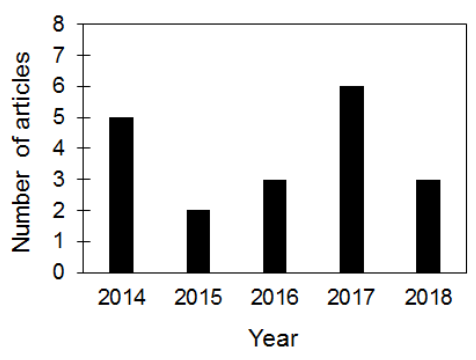

Fig. 1. Number of articles published regarding GO-modified membrane for VRFB application based on Web of Science and Scopus search engines (topic keywords are "graphene", "membrane", and "vanadium redox").

\section{GO-modified membranes for VRFB}

Composite membranes with GO and its derivatives have become one of the best approaches to overcome the issues of vanadium ion selectivity of the membrane in VRFB. Nafion membrane undoubtedly has supreme chemical and stability towards vanadium electrolytes [23]. Although it has excellent stability, this membrane suffers a lot from very high vanadium permeability. This is because the membrane's hydrophilic side formed by the sulfonic acid group not only promotes proton movement but also vanadium ion. Hence, most of the research have been focused on modifying Nafion by GO to form a composite membrane to enhance selectivity.

In 2014, GO was used as a vanadium barrier for Nafion 117 for the first time [24]. In this work, pristine Nafion 117 was coated side-by-side with a GO emulsion solution. It was revealed that vanadium permeability of GO-Nafion $\left(6.1 \times 10^{-7} \mathrm{~cm}^{2} \mathrm{~min}^{-1}\right)$ reduced significantly compared to pristine Nafion $117\left(20.5 \times 10^{-7} \mathrm{~cm}^{2} \mathrm{~min}^{-1}\right)$. Consequently, by modifying the membrane with GO layer, the CE and energy efficiency (EE) of the VRFB system was successfully improved.

In another study, the group also carried out research on the effect of GO-membrane composition on vanadium permeability [25]. The composite membrane in this research was prepared through a simple technique, which was casting method with different GOloadings $(0.001 \mathrm{wt}$ \% $\%$ to $1 \mathrm{wt}$ \%). From the study, it was found that the decrease of vanadium permeability was linearly related to the increase in GO loading. In contrast, the proton conductivity of composite membranes decreased as GO loading increased. This proves that the difference in GO loading promotes different membrane microstructures, which increases the inter-planar space dimension of the membrane and consequently blocks vanadium permeation along with proton transfer. Due to this reason, the final GO loading was optimised to $0.01 \mathrm{wt}$ \% since the membrane possessed the highest $\mathrm{EE}$ of $82.5 \%$ at $40 \mathrm{~mA} \mathrm{~cm}$. Figure 2 displays the distribution of GO with different weight percentages on the membrane samples. 


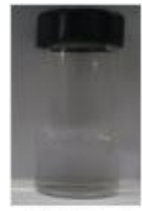

Go Owt\% soln.

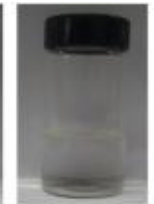

$600001 w+\%$ soln

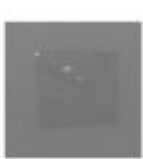

60000010

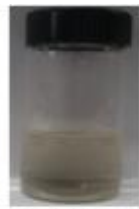

600.01 wts soln

(a)

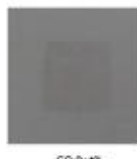

Go ontis

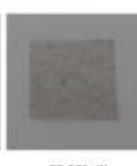

(b)

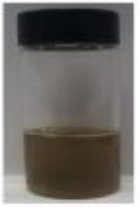

Go $0.1 w t \%$ soln.

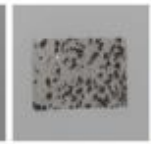

colime

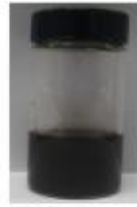

Go 1wts soin.

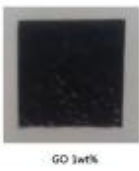

Fig. 2. GO/Nafion membranes with different GO-loadings [25].

To gain more understanding regarding vanadium ion transport process in GOmodified membrane, the mechanism of proton and vanadium ion transport process in the membrane matrix was proposed. Similarly, Yu et al. [26] incorporated Nafion matrix and GO through casting method. In their work, Nafion and GO were mixed and casted to form Nafion/GO (rNafion/GO). The use of $\mathrm{GO}$ in the recast Nafion successfully proved that GO can act as an outstanding physical barrier to hinder vanadium transport by reducing the travelling path of $\mathrm{VO}^{2+}$. This is because prior to the addition of $\mathrm{GO}$ as a filler in the membrane matrix, $\mathrm{VO}^{2+}$ can transfer freely through water channels. When GO is added, the water channels of the composite membrane shrunk due to the interaction between sulfonated acid groups in the Nafion matrix and oxygen-containing groups in GO. Hence, the randomly distributed GO tortures the water channels that obstruct the movement of $\mathrm{VO}^{2+}$ and proton ions. As a result, vanadium permeability of the fabricated membrane is reduced by half with a slight decrease in proton conductivity value. The proposed proton and vanadium ion transport mechanisms in rNafion/GO are shown in Figure 3.

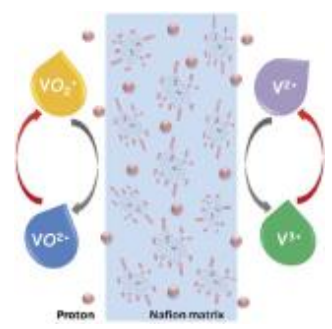

Fig. 3. Proposed mechanisms for vanadium ion permeation and proton transport process in rNafion/GO composite membrane [26].

Although it has been proven that Nafion/GO composite membrane prepared by solution casting can successfully reduce vanadium permeability, randomly dispersed $\mathrm{GO}$ has a limited role of GO as a vanadium ion barrier. Not only that, often when GO is used in membrane fabrication as a modifier, the proton conductivity value is also sacrificed due to the impeded water channels of Nafion matrix. Therefore, a more orientated Nafion/GO composite membrane is preferred to provide a greater effect on vanadium barrier and minimise reduction in proton conductivity value. An interesting 
work was done by $\mathrm{Su}$ et al. [27] whom prepared an orientated Nafion/GO composite with ultra-thin (40-400 nm) spin-coating layer. The vanadium barrier effect of Nafion/GO was maximised by the aligned arrangement of GO nanosheets parallel to Nafion surface. By comparing the results between the disorientated and orientated Nafion/GO, the orientated composite shows much lower vanadium permeability with better ionic conductivity. Table 1 lists the results for Nafion/GO composite membranes.

Table 1. Selectivity ratio of ionic conductivity to vanadium permeability for various membranes [27].

\begin{tabular}{|c|c|c|c|c|c|}
\hline Samples & $\begin{array}{c}\text { Thickness of } \\
\text { Membrane } \\
(\text { wet, } \mu \mathrm{m})\end{array}$ & $\begin{array}{l}\text { Thickness of } \\
\text { Recast Layer } \\
(\mu \mathrm{m})\end{array}$ & $\begin{array}{c}\text { Proton } \\
\text { Conductivity } \\
\left(\mathrm{mS} \mathrm{cm} \mathbf{c m}^{-1}\right)\end{array}$ & $\begin{array}{l}\text { Permeability } \\
\left(10^{-7} \mathrm{~cm}^{2} \text { min }^{-}\right. \\
1)\end{array}$ & $\begin{array}{c}\text { Selectivity } \\
\left(10^{7} \mathrm{mS} \text { min }\right. \\
\left.\mathrm{cm}^{-3}\right)\end{array}$ \\
\hline N212 & 58.0 & - & 76.3 & 28.0 & 2.7 \\
\hline $\mathrm{P}-\mathrm{N}^{\mathrm{a}}$ & 58.3 & - & 75.2 & 31.3 & 2.4 \\
\hline $\mathrm{M}-0.1^{\mathrm{a}}$ & 57.5 & 0.40 & 74.9 & 1.5 & 50.0 \\
\hline$M-2^{\mathrm{a}}$ & 57.4 & 0.44 & 72.0 & 0.82 & 87.7 \\
\hline GO-N ${ }^{b}$ & 210.0 & 10.00 & 64.0 & 6.1 & 10.5 \\
\hline $\mathrm{rN} / \mathrm{GO}^{\mathrm{c}}$ & 70 & - & 29.3 & 8.0 & 3.7 \\
\hline $\mathrm{GO} / \mathrm{N}-0.01 \%^{\mathrm{d}}$ & 27.8 & - & 32.0 & 5.1 & 6.3 \\
\hline
\end{tabular}

${ }^{a} \mathrm{P}-\mathrm{N}$ refers to pristine Nafion, whereas $M-0.1\left(0.1 \mathrm{mg} \mathrm{g}^{-1}\right)$ and $\mathrm{M}-2\left(2 \mathrm{mg} \mathrm{g}^{-1}\right)$ refer to the concentrations of $G O$ in the spin-coating suspension.

${ }^{b}$ Disorientated GO/Nafion coating membrane prepared by Shul et. al by immersing Nafion membranes in $5 \mathrm{mg} \mathrm{g}^{-1}$ GO/Nafion suspension [24].

${ }^{c}$ Disorientated $r N a f i o n / G O$ composite membrane prepared by $Y u$ et al. using solution casting technique [26].

${ }^{d}$ Disorientated GO/Nafion blend membrane (0.01\%) prepared by Lee et. al using casting technique [25].

Other than Nafion, sulfonated aromatic polymers or non-perfluorinated polymers can also be considered as one of the potential membranes for VRFB applications. For instance, sulfonated poly(ether ether ketone) (SPEEK) [28, 29], sulfonated poly(arylene ether ketone) [30], and sulfonated polyimide (SPI) [23, 31] have gained interest from researchers to act as membranes in VRFB due to their high ionic conductivity, good mechanical properties, and lower cost compared to Nafion [29, 32, 33]. In the case of sulfonated aromatic polymers, the degree of sulfonation (DS) determines the properties of each membrane. In general, proton conductivity of membrane is higher for the membrane with higher DS [34]. Despite its high conductivity at high DS, the membrane usually exhibits high vanadium permeability and poor mechanical properties $[35,36]$. Therefore, besides research on modifying Nafion, non-perfluorinated membranes are also considered for the fabrication of composite membranes with GO-based materials.

Dai et al. [34] demonstrated the incorporation of GO into pristine SPEEK via conventional solution casting method. The DS selected for SPEEK was 0.75 as the work aimed to study the effect of vanadium barrier properties and mechanical properties of SPEEK membrane with high DS. By comparing Nafion to SPEEK, the SPEEK membrane has a lower ratio of hydrophilic/hydrophobic separation. This is because the backbone of SPEEK membrane is less hydrophobic, whereas the sulfonic acid group attached to the SPEEK structure is less acidic [37, 38]. Hence, SPEEK possesses narrower and more branched filled water channels. The incorporation of impermeable 2D-layered GO nanosheet with sulfonic acid group lowers vanadium ion permeability 
since a significant increase in the tortuosity of membrane structure twists the diffusion path of vanadium ions.

To study the stability of S/GO membrane in VRFB testing, a cycling test was carried at $80 \mathrm{~mA} \mathrm{~cm}$. The results from VRFB testing are displayed in Figure 4. As shown in the figure, the $\mathrm{CE}$ and $\mathrm{VE}$ of $\mathrm{S} / \mathrm{GO}$ membrane are higher than Nafion membrane with no obvious decline even after 300 cycles. This online VRFB testing further confirmed the effectiveness of GO as the modifier in SPEEK membrane. The S/GO membrane also managed to retain almost half of its charge capacity (56.3\%) even at 300 cycles. This clearly indicates that GO does not only help SPEEK to suppress vanadium permeation but also enhances the stability of the composite membrane in a severe VRFB environment.
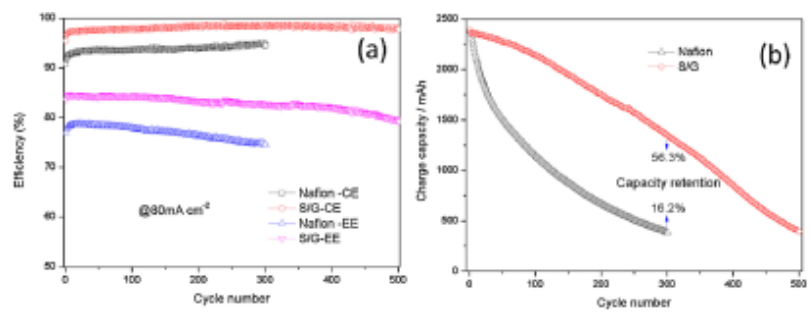

Fig. 4. VRFB cycling performance and charge capacity with S/GO and Nafion membranes [34].

Analysis of the performance and stability of S/GO was further studied in detail by varying GO content (i.e., with 1, 2, 3 or 5 wt. \%) [39]. The composite membrane in this work was denoted as $\mathrm{S} / \mathrm{GO} X$, where $X$ represented the GO content. Ion selectivity is the ratio of proton conductivity to vanadium ion permeability of the membrane. In VRFB, ion selectivity is one of the trickiest parameters when selecting a suitable membrane since it is very difficult to balance proton conductivity and vanadium permeability of the membrane. From this work, it was determined that S/GO 2 and S/GO 3 membranes

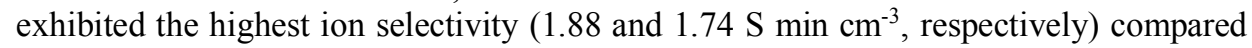
to the pristine SPEEK and S/GO 5 membranes (1.47 and $1.44 \mathrm{~S} \mathrm{~min} \mathrm{~cm}^{-3}$, respectively). Thus, the optimum GO content for SPEEK membrane was set at 2 and $3 \mathrm{wt}$. $\%$.

Based on the physicochemical properties of the composite membranes, it was revealed that even a small amount of GO could enhance the mechanical properties of the membrane. By comparing the pristine SPEEK and S/GO composite membranes, the latter possessed higher breaking strength. The breaking strength of the composite membrane showed a linear relationship with GO content. The elongation percentage of S/GO 2 and S/GO 3 membranes was also lower than the pristine Nafion and SPEEK membranes. The reason for the improvement of the mechanical properties is because of the strong hydrogen bond between SPEEK and GO. This shows that the membranes are tough enough to be used in VRFB. Table 2 provides the data of mechanical properties for the studied membranes. Another point to note is that the cyclability of the composite membrane also improved as the result of mechanical properties enhancement. Similar to the previous work [34], when tested with a VRFB single cell, the membrane survived in a long continuous run (300 cycles) without obvious erratic behaviour as compared to pristine SPEEK and Nafion membranes as shown in Figure 5. 
Table 2. Mechanical properties of the investigated membranes.

\begin{tabular}{lcc}
\hline Samples & $\begin{array}{c}\text { Breaking } \\
\text { Strength } \\
\text { (MPa) }\end{array}$ & $\begin{array}{c}\text { Elongation } \\
\text { Percentage } \\
\text { (\%) }\end{array}$ \\
\hline Nafion 117 & 21.4 & 218 \\
SPEEK & 16.7 & 86 \\
S/GO 2 & 28.8 & 83 \\
S/GO 3 & 31.4 & 82 \\
\hline
\end{tabular}

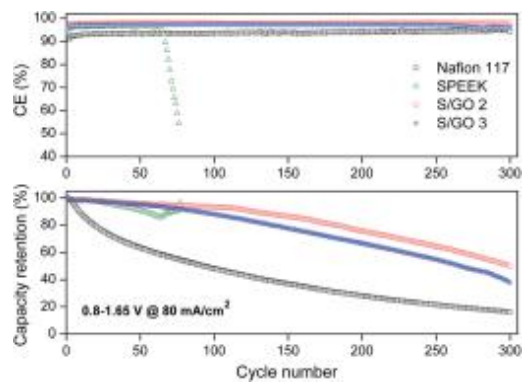

Fig. 5. Cycle performance of Nafion 117, pristine SPEEK, and S/GO membranes for different GO contents [39].

In another work, zwitterionic polymer-functionalised GO was introduced into SPI matrix [40]. Zwitterionic polymer is a polymer that contains both cationic and anionic groups. The use of zwitterionic polymer as an excellent vanadium barrier and for improving membrane stability was proven by Yi et al. [41]. Interestingly, this approach shows promising results as the influence of GO and zwitterionic polymer helps in reducing vanadium permeability and simultaneously increases proton conductivity. This effect was realised as a result of the dual coordination influence between GO and zwitterionic polymers. This is because the acid-base interaction between the quaternary ammonium groups from the zwitterionic and sulfonic acid groups from SPI creates proton transport pathways in the interfacial zone between the SPI matrix and GO filler. As a consequence, the membrane possesses balanced proton and vanadium permeability properties. Figure 6 shows the prepared composite membrane of zwitterionic polymerfunctionalised GO.

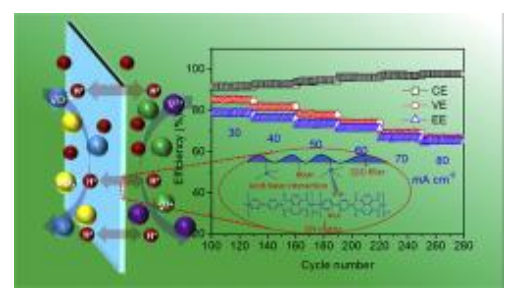


Fig. 6. Zwitterionic polymer-functionalised GO and its efficiency at various current densities [40].

Although vanadium properties and the stability of GO-modified membranes for VRFB could be improved, it is important to widen the study for the role of GO in the improvement of vanadium permeability. More efforts should also be taken to study various methods for the fabrication of $\mathrm{GO}$ as a modifier for membranes with high ion selectivity and long-term operation stability.

\section{Conclusion}

In summary, GO-modified membranes can be employed as vanadium redox flow membranes to reduce vanadium permeability. In addition, the use of GO as a modifier can also help to strengthen the chemical and mechanical stability of the composite membrane. Finally, the flexibility of GO-modified membrane, as well as its low cost, makes it easier to be commercialised at a large scale. Further research and investigations are encouraged as GO is now considered as one of the emerging building materials for VRFB application.

The research was primarily supported by Universiti Teknologi Malaysia and the Ministry of Higher Education under Fundamental Grant (Vot. No. 4C116) and a joint partnership research programme of Innovative Research Universities of Australia and Malaysia Research University Network (IRU-MRUN) (Vot. No. 00M84).

\section{References}

1. A. Cunha, J. Martins, N. Rodrigues, and F. P. Brito, Int. J. Energ. Res. 39, 889-918 (2015).

2. H. Prifti, A. Parasuraman, S. Winardi, T. M. Lim, and M. Skyllas-Kazacos, Membranes (Basel) 2, 275-306 (2012).

3. A. Parasuraman, T. M. Lim, C. Menictas, and M. Skyllas-Kazacos, Electrochim. Acta 101, 27-40 (2013).

4. A. Z. Weber, M. M. Mench, J. P. Meyers, P. N. Ross, J. T. Gostick, and Q. H. Liu, J.Appl. Electrochem. 41, 1137-1164 (2011).

5. H. S. Chen, T. N. Cong, W. Yang, C. Q. Tan, Y. L. Li, Y. and L. Ding, Prog. Nat. Sci. 19, 291-312 (2009).

6. M. Etesami et al., Int. J. Hydrog. Energy 43, 189-197 (2018).

7. R. Ye et al., J. Electrochem. En. Conv. Stor. 15, (2017).

8. S. Maurya, S.-H. Shin, Y. Kim, and S.-H. Moon, RSC Adv. 5, (2015).

9. X. Li, H. Zhang, Z. Mai, H. Zhang, and I. Vankelecom, Energy Environ. Sci. 4, 1147 (2011).

10. C. Minke and T. Turek, J. Power Sources 286, 247-257 (2015).

11. R. R. Nair, H. A. Wu, P. N. Jayaram, I. V. Grigorieva, and A. K. Geim, Science 335, 442-4 (2012).

12. H. W. Kim et al., Science 342, 91-95 (2013).

13. H. Li et al., Science 342, 95-98 (2013).

14. M. Hu and B. Mi, Environ. Sci. Technol. 47, 3715-23 (2013). 
15. W. Gao et al., Angew. Chem. Int. Ed. Engl. 53, 3588-93 (2014).

16. E. Abouzari-Lotf, M. Etesami, and M. M. Nasef, Eds, (2018).

17. J. Ma, D. Ping, and X. Dong, Membranes 7, (2017).

18. N. Mahmood, C. Zhang, H. Yin, and Y. Hou, J. Mater. Chem. A, 2, (2014).

19. R. K. Joshi, S. Alwarappan, M. Yoshimura, V. Sahajwalla, and Y. Nishina, Applied Materials Today, 1, 1-12 (2015).

20. M. F. De Volder, S. H. Tawfick, R. H. Baughman, and A. J. Hart, Science, 339, 535-9 (2013).

21. D. R. Paul, Science, 335, 413-4 (2012).

22. H. Huang, Y. Ying, and X. Peng, J. Mater. Chem. A, 2, 13772-13782 (2014).

23. Y. Zhang et al., J. Mater. Sci. 53, 14506-14524 (2018).

24. Y. G. Shul and Y. H. Chu, Sci. Adv. Mater. 6, 1445-1452 (2014).

25. K. J. Lee and Y. H. Chu, Vacuum 107, 269-276 (2014).

26. L. Yu, F. Lin, L. Xu, and J. Xi, RSC Adv. 6, 3756-3763 (2016).

27. L. Su, D. Zhang, S. Peng, X. Wu, Y. Luo, and G. He, Int. J. Hydrog. Energy 42, 21806-21816 (2017).

28. Z. Li, W. Dai, L. Yu, J. Xi, X. Qiu, and L. Chen, J. Power Sources 257, 221-229 (2014).

29. Y. Ji, Z. Y. Tay, and S. F. Y. Li, J. Membr. Sci. 539, 197-205 (2017).

30. S. Yang, Y. Ahn, and D. Kim, J. Mater. Chem. A 5, 2261-2270 (2017).

31. Y. Pu et al., Appl. Surf. Sci. 448, 186-202 (2018).

32. D. H. Hyeon, J. H. Chun, C. H. Lee, H. C. Jung, and S. H. Kim, Korean J. Chem. Eng. 32, 1554-1563 (2015).

33. S. Kim et al., J. Appl. Electrochem. 41, 1201-1213 (2011).

34. W. Dai et al., Electrochim. Acta 132, 200-207 (2014).

35. N. Wang, S. Peng, H. Wang, Y. Li, S. Liu, and Y. Liu, Electrochem. Commun. 17, 3033 (2012).

36. S. Ren et al., J. Membr. Sci. 247, 59-63 (2005).

37. Z. L. Li et al., Free Radic Biol. Med. 57, 132-40 (2013).

38. Y. Heo, H. Im, and J. Kim, J. Membr. Sci. 425, 11-22 (2013).

39. W. Dai, Y. Shen, Z. Li, L. Yu, J. Xi, and X. Qiu, J. Mater. Chem. A 2, 12423-12432 (2014).

40. L. Cao, L. Kong, L. Kong, X. Zhang, and H. Shi, J. Power Sources 299, 255-265 (2015).

41. Y. Li, X. Lin, L. Wu, C. Jiang, M. M. Hossain, and T. Xu, J. Membr. Sci. 483, 60-69 (2015). 